how to plan and use to the best advantage large equipment? also how to provide the minimal necessary influx of young physicists, to avoid missing a generation of potential physicists, and creating a generation gap? how to keep the enthusiasm and efficiency of the many physicists recruited in the sixties?

Another top priority is to help marry physics with Society. We should favour contacts of physics with other branches of science: why for instance has the division on biophysics and physics in medicine, discussed at York, never materialized? We should worry how physics teachers are trained and kept in contact with our evolving science and technology. We should explore employment outside the universities for young and older physicists. We should keep the gates fully open between fundamental and applied research, between university, major organisms of research and industry, small and large.

For the universities, this implies a new look at training - quantity and quality, the flux and ages of PhD's, interest in questions arising from industry or injection into industry of various knowledge and skills. For industry, this means a will to develop contacts with the universities and extract a maximum advantage in stimulation and sometimes - collaboration. EPS has here taken some steps in the right direction, despite the difficulties due to the splitting of Europe into different nations and economic systems. The action should be encouraged, not only by a support of ACAPPI and the Physics and Society Committees, but, for instance, by having more industrialists in our Council, taking more advice from our Associate members, fostering contacts on a European scale of various national societies of applied science.

Finally we must care for good relations within Europe as well as with the rest of the world.

Within Europe, there are obvious and very real problems between east and west, which can be overcome only by a constant effort of mutual understanding and respect, although we must acknowledge that they could become intractable as a result of an international crisis. Some Secretariat activity in say Budapest might lead to a more balanced activity. There are also subtle but serious differences between north and south. Some effort should be made to help countries where physics is not a top priority to make it better known and, perhaps, better taught.

UNESCO and the ICTP in Trieste, the national physical societies, large scientific museums, large research institutes could be asked to help, in developing say, summer schools adapted for physics teachers, TV programmes or travelling physics exhibitions, cutting across national borders. Major progress towards the unity and effectiveness of EPS would come from the granting of financial support to enable members of committees to attend meetings.

But Europe must also look outside its boundaries. A first step towards APS should be followed by others towards countries such as Japan or Australia. An exchange scheme for young post-doctorates outside Europe could possibly be pushed through for developing countries, together with the European Science Foundation.

In these activities, EPS should keep in mind two considerations:

- EPS is not alone in Europe. When it was created, there were many national physical societies, which are now all members of EPS. I do not think that this major difference with APS should be considered in any way as a weakness of EPS. It is a source of richness and possibly strength for physics in Europe. Indeed, the creation of EPS has stimulated the national societies, and such questions as regional implantation, budgets, creation of research posts, teaching of physics and jobs for physicists can mainly be fought for and im- plemented at the national level - for the present at least. What EPS can do in these fields is to help and create a common will and common attitudes, while the more active the national societies are in these areas, the healthier will physics be in Europe.

- EPS is fundamentally short of money. It cannot ask too much from its members, who themselves have limited means, but it has to make ends meet and therefore it cannot, at the present, launch any new commitment that would involve expense. Indeed our budget has been in deficit for several years, and we just cannot go on in this way. If we want to have an effective action, we cannot restrict our commitments any more. But the time seems ripe for a renewed determination to help ourselves. We must, each of us, try and attract new individual members, as our retiring President has done so effectively. We must attract new associate members: organisms such as Nordita, CNRS, SERC and their eastern counterparts should be brought in. But lasting success requires that our supporters keep their interest: our future is in our own hands.

\title{
The Quantum Hall Effect
}

\section{$\mathrm{K}$. von Klitzing, Munich \\ (Technical University)}

1982 Hewlett-Packard Prize Winner

When Hall effect measurements are reported, sensational results are not as a rule expected. Normally the effect is used to measure the concentration of free electrons in semiconductors and uncertainties of about $10 \%$ are typical in such experiments.

The peculiarity of the quantum Hall effect is that it is highly reproducible and the values measured are independent of any experimental parameter. Measurements in Germany, Japan, USA, and Switzerland on different materials and different samples, with different geometries, yield data which are identical within the ex- perimental uncertainty of $10^{-6}$. It seems that the result depends exclusively on the fundamental constants $h$ (Planck's constant) and $e$ (elementary charge). At present all experimental results and theoretical investigations indicate that on the basis of the quantum Hall effect, a resistor can be fabricated with a fixed resistance value of: $h / e^{2} \cong 25812.8 \Omega$ (or an integer part of this).

As the electrical unit ohm can be established with an uncertainty of $10^{-7}$ on the basis of the definitions of the mechanical and electrical units kilogram, metre, second, and ampere (SI units), the quantum

Fig. 1 - The former President of EPS, Professor A.R. Mackintosh presenting the 1982 HewlettPackard prize to Professor K. von Klitzing (right). Looking on are (left) Mr. J.L. Doyle, VicePresident, Research and Development, of Hewlett-Packard, (centre) Sir Sam Edwards, Chairman of the Prize Committee and (largely hidden) Professor J. Devreese, Chairman of the Condensed Matter Division. The presentation was made during the 2nd CMD General Conference in Manchester.

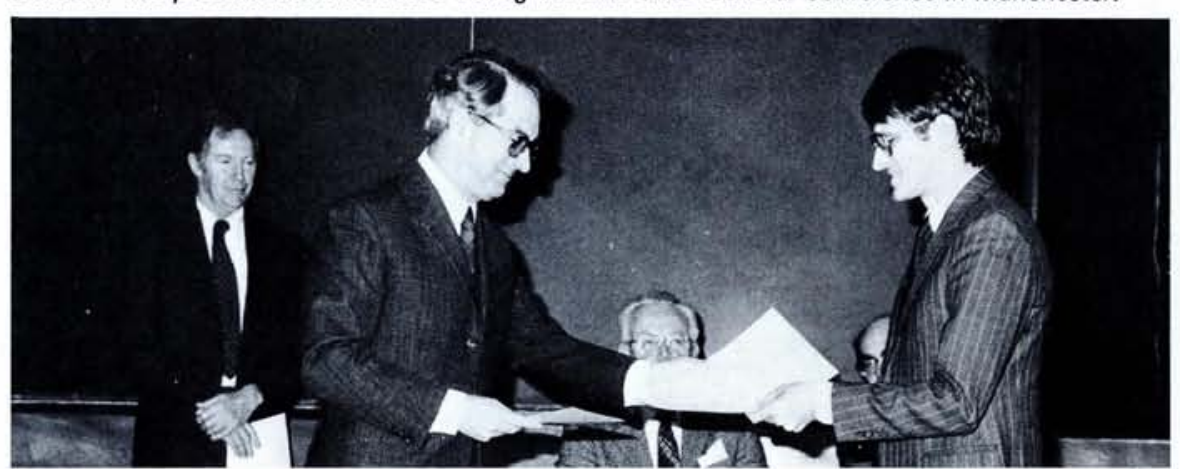




\section{Citation:}

"In a two-dimensional electron gas subject to a strong magnetic field applied perpendicularly, the energy spectrum becomes discrete at low temperatures. The inversion layer occurring in Metal Oxide Semiconductor Field Effect Transistors in an example of such a twodimensional system. You recognized the attractiveness of this configuration for metrological applications, well in advance of an appropriate theoretical foundation."

Hall effect can be used to determine the value of the fundamental constant $h / e^{2}$ with an uncertainty down to $10^{-7}$, by comparing the value $R_{H}=h / e^{2}$ of the quantized Hall resistance with a standard resistor calibrated in SI ohms. As the accuracy of the fine-structure constant $\alpha$ depends exclusively on the value of $h / e^{2}$, the quantum Hall effect has been used to determine the fine-structure constant with an accuracy comparable with the best values obtained by other methods. Experimental data are:

$$
\begin{aligned}
& \alpha^{-1}=137.03592 \pm 0.00008 \text { (F.R.G.) } \\
& \alpha^{-1}=137.03589 \pm 0.00012 \text { (Japan) } \\
& \alpha^{-1}=137.035968 \pm 0.000023 \text { (USA) }
\end{aligned}
$$

The officially recommended value is:

$$
\alpha^{-1}=137.03604 \pm 0.00011 \text {. }
$$

The uncertainties in the value of $\alpha$ when determined by the quantum Hall effect arise partly from the instabilities in the value of the calibrated reference resistor, so that different national standards laboratories are investigating the possibility of using the quantum Hall resistance as a time independent reference resistor in order to look for drifts and instabilities in the value of the maintained standard ohm.

This application of the quantum Hall effect is related to a similar application of the a.c. Josephson effect which combines the fundamental constant $h / e$ with the electrical unit volt, and has consequently been used for high precision measurements of the fundamental constant $h / e$. The voltage produced by the a.c. Josephson effect is more stable and reproducible than the maintained voltage unit using standard cells, so that accurate voltage measurements are expressed at present in units of the Josephson-Volt using a defined value for $h / e$. The same may happen with the quantum Hall effect. If the experimental uncertainty in the measurements of the quantized Hall resistance $R_{H}=h / e^{2}$ can be reduced below $10^{-7}$, the quantized Hall resistance could be used as a standard resistor with a resistance value based on an internationally defined value for $h / e^{2}$.

First of all however, it must be proved that the quantized Hall resistance $R_{H}$ depends indeed exclusively on the fundamental constant $h / e^{2}$. Up to now, the experiments demonstrate that within the experimental uncertainty, $R_{H}$ agrees with $h / e^{2}$, but this is not a proof that this will be correct at a higher level of accuracy. From the theoretical point of view, the value of
$R_{H}$ can readily be derived on the basis of an idealized model (see below), but even today, two years after the discovery of the quantum Hall effect, no microscopic transport theory exists, which can predict at which level of accuracy, corrections to $R_{\mathrm{H}}$ $=h / e^{2}$ are to be expected.

\section{Hall Effect}

The measured quantity in Hall effect experiments is the Hall voltage $U_{H}$, which is proportional to the Hall field $E_{\text {v }}$ built up in a direction perpendicular to both the current direction $j_{x}$ of charged particles and the transverse magnetic field $B_{2}$ across which they are moving. Classically, the Hall field $E_{v}$ can be deduced from the equilibrium between the force on a moving electron in a magnetic field (Lorentz-force) and the force on the electron in the Hall field and is given by the expression:

$$
E_{y}=\left(B_{z} n_{\mathrm{e}} e\right) j_{x} \equiv \rho_{x y} j_{x}
$$

where $n_{\mathrm{e}}$ is the electron density, $j_{x}$ is the current density, which has for devices with Hall geometry only one component in the direction of the long axis of the sample, and $\rho_{x y}$ is the off-diagonal component of the resistivity tensor. Later it will be shown that $\rho_{x y}$ is identical to the Hall resistance $R_{\mathrm{H}}$. For measurements of the quantum Hall effect it is essential that the energy spectrum of the electrons, which contribute to the Hall effect, becomes fully quantized into well separated energy levels.

Theoretically, the energy spectrum

$$
E=\hbar^{2} k_{x}^{2} / 2 m+\hbar^{2} k_{y}^{2} / 2 m+\hbar^{2} k_{z}^{2} / 2 m
$$

of conduction electrons in a semiconductor with an effective mass $m$ is already discrete, because the wave vector $k$ is quantized (an integer of the de Broglie wavelength $\lambda=2 \pi / k$ has to fit into the sample). However, for a sample of macroscopic dimensions, the energy separation between adjacent energy levels is too small to be resolved, so that a quasi continuous energy spectrum is observed. A discrete energy spectrum can however be obtained, if the effective dimensions of the sample are reduced. This can be realized in a socalled two-dimensional electron gas (conducting layer with a thickness of some nanometres) placed in a strong transverse magnetic field.

The magnetic field $B_{z^{\prime}}$ "localizes" the electron in the $x y$-plane within the area of the cyclotron orbit (the diameter of the ground state cyclotron orbit is about $16 \mathrm{~nm}$ at $B_{z}=10 \mathrm{~T}$ ), resulting in a well separated energy spectrum for the motion of the electrons in the plane perpendicular to the magnetic field:

$$
E_{\|}=(n+1 / 2) \hbar \omega_{c} \quad n=0,1,2, \ldots
$$

$\omega_{c}=e B_{z} / m$ for the cyclotron frequency.

Each of these energy levels has a degeneracy factor per unit area of :

$$
N=e B_{z} / h
$$

corresponding to the number of cyclotron orbits per unit area. The confinement of the

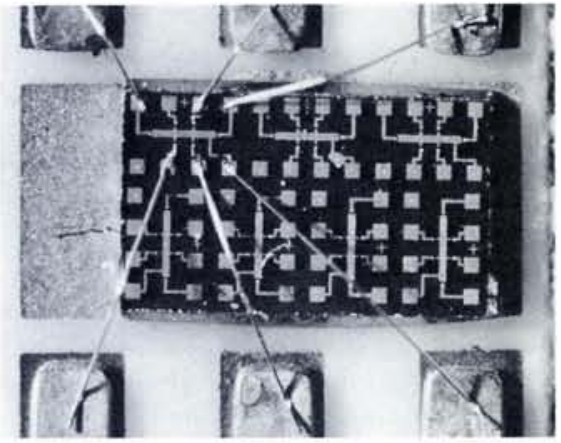

Fig. 2 - Silicon MOS devices for measurements of the quantized Hall resistance. The length of one device is $0.4 \mathrm{~mm}$.

electrons within the thin layer leads additionally to well separated energy levels $E$ for the motion of the electron in the third dimension (z-direction), so that the total energy can be written:

$$
E_{j, n}=E_{j}+(n+1 / 2) \hbar \omega_{c}
$$

The energy difference between adjacent levels is of the order of $10 \mathrm{meV}$ in a magnetic field of $10 \mathrm{~T}$.

Such a discrete energy spectrum with energy gaps larger than all other relevant energies (thermal energy, level broadening) is necessary for the observation of the quantum Hall effect because only when an integer number $i$ of energy levels $E_{j n}$ (eq. 4) are occupied with electrons while all other levels at higher energies are empty, does the measured quantity $\rho_{x y}$ in the Hall effect become a universal constant

$$
\rho_{x y}=B_{z} / n e=h / e^{2} i \quad \mathrm{i}=1,2,3, \ldots
$$

This result can easily be deduced from eq. (3), if the carrier density $n_{e}$ for fully occupied energy levels is set equal to:

$$
n_{\mathrm{e}}=i N=i e B_{z} / h
$$

\section{Faculty Positions} The Department of MATERIALS SCIENCE AND ENGINEERING at SUNY STONY BROOK invites applications for two anticipated tenure-track assistant professorships. Candidates with more extensive experience will be considered for positions for higher rank. Candidates must have a Ph.D. degree in physical sciences or engineering with experience in ELECTRONIC MATERIALS, and/or STRUCTURAL MATERIALS. Research interest in synchrotron techniques will be an advantage. Expected starting date is September 1982.

Applications should be sent to:

Professor F.F.Y. Wang,

Chairman of the Search Committee, DEPT. OF MATERIALS SCIENCE \& ENGINEERING,

SUNY STONY BROOK, Stony Brook, NY 11794.

SUNY Stony Brook is an equal opportunity/affirmative action employer. $\mathrm{AK}=340 \mathrm{E}$. 
For a two-dimensional electron gas, it is natural to define $j_{x}$ as the current density per unit width. The resistivity component $\rho_{x y}$ is then identical to the Hall resistance $R_{H}^{x y}$ (Hall voltage $U_{H}$ divided by the current $/$ ) because the ratio $E_{y} / j_{x}$ in eq. (1) is identical to the measurable quantity $R_{H}=U_{H} / /$. Generally, all Hall effect measurements on two-dimensional electron systems in a strong magnetic field should yield Hall resistance values $R_{H}=U_{H} / /=h / e^{2} \mathrm{i}$, as long as exactly $i$ energy levels $E_{n j}$ are occupied with electrons. This condition, that precisely an integer number of energy levels contribute to the Hall effect is fulfilled, if the elastic scattering rate for electrons vanishes, because the gap separating occupied and empty states then prevents energy-conserving transitions. No scattering means that a supercurrent $j_{x}$ flows perpendicular to the electric field $E_{y}$ and magnetic field $B_{z^{\prime}}$ as expressed by the condition $\rho_{\mathrm{xx}}=0$ and $\sigma_{\mathrm{xx}}=0$ for the diagonal element of the resistivity and conductivity tensor.

The confusing statement that the resistivity $\rho_{x x}$ and the conductivity $\sigma_{x x}$ both become zero originates from the condition for the tensor product $\rho . \sigma=1$ which leads to the equation $\sigma_{x x}=\rho_{x x} / \rho_{x y}^{2}+\rho_{x x}^{2}$. With no magnetic field $\left(\rho_{x y}=0\right)$ the well-known condition $\sigma_{x x}=1 / \rho_{x x}$ is obtained, but with strong magnetic fields $\left(\rho_{x y} \gg \rho_{x x}\right)$ the tensor components $\rho_{x x}$ and $\sigma_{x x}$ are directly proportional. The significance of $\sigma_{x x}=0$ is that no current transport in the direction of the electric field is possible which is true for non-interacting electrons in a magnetic field, because the motion is perpendicular to the electric field. Experimentally, the situation $\rho_{x x}=0$ corresponds to a vanishingly small voltage drop $U_{p}$ in the direction of the electric current.

\section{Two-Dimensional Electron Gas}

For measurements of the quantized Hall resistance, a two-dimensional electron gas (2DEG) is needed. As long as 16 years ago, Fang and Howard demonstrated that such a 2DEG exists at the semiconductor surface of a silicon field effect transistor. This device is basically a metal-oxide-semiconductor (MOS) capacitor in which electrons can be induced at the surface of a p-type semiconductor, if a sufficiently high positive gate voltage is applied to the metal side of the capacitor. Calculations shows that the induced electrons are confined within such a narrow channel close to the semiconductor surface, that quantization effects become important and an energy spectrum typical for a 2DEG is produced at low temperatures. Electrical contacts on the surface allow measurements of transport phenomena. Fig. 2 shows a top view of such a device with two current contacts at the ends of the long axis (the dimension of the sample in the surface plane is determined by the rectangular geometry of the

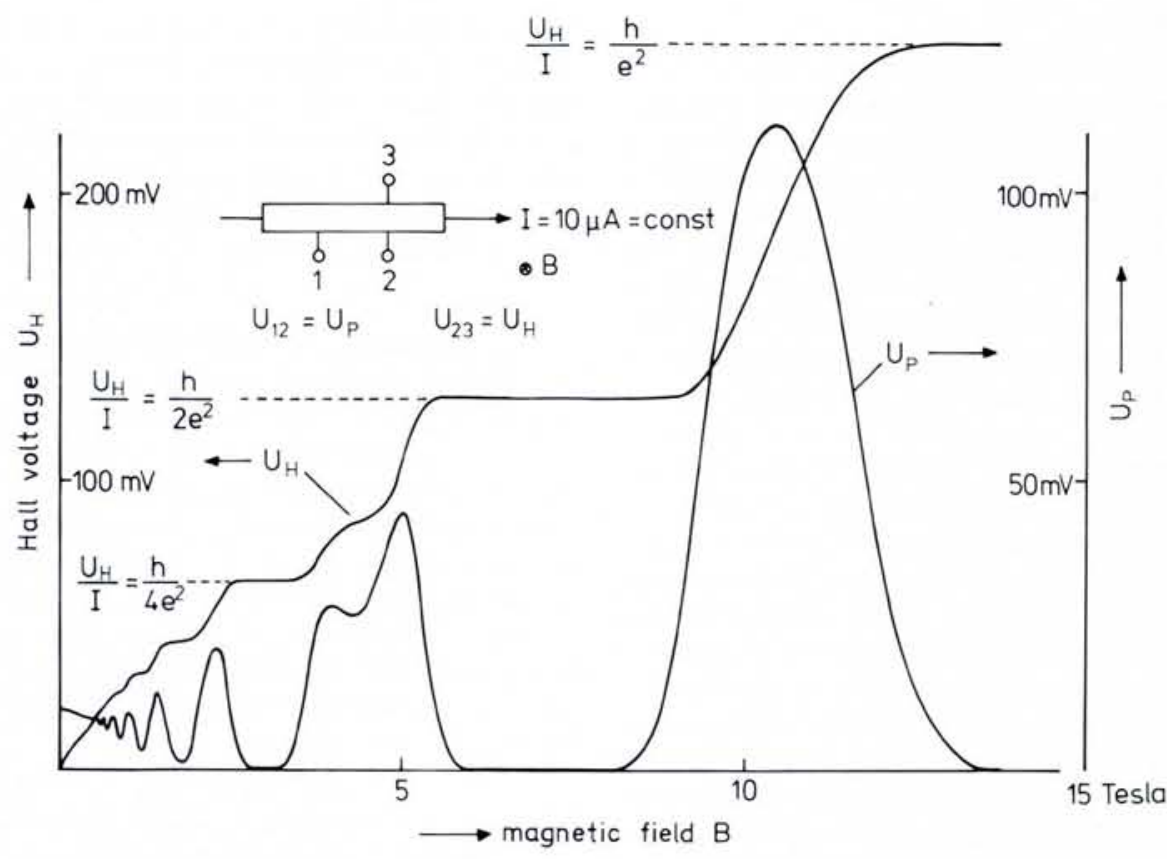

Fig. 3 - Hall voltage $\left(U_{H}\right)$ and resistivity $\left(\rho_{x x} \sim U_{\rho}\right)$ data for a GaAs-AI 0.3 Ga $a_{0.7}$ As heterostructure as a function of the magnetic field $B$ at a temperature of $T=1.6 \mathrm{~K}$. Steps in the Hall voltage are visible with resistance values $R_{H}=h / e^{2} i$ at magnetic fields where $U_{p}$ becomes zero.

gate metal) and four potential probes for resistivity measurements along the surface channel and Hall effect measurements across the channel. The carrier density and therefore the conductivity in the surface channel can be changed by means of the gate voltage.

A two-dimensional electron gas can also be formed at the interface between two semiconductors, e.g. GaAs and $\mathrm{Al}_{0.3} \mathrm{Ga}_{0.7}$ As. In this case, the narrow potential well for the 2DEG originates from the differences in the energy gaps and work functions of the two semiconductors. The electron concentration in the interface channel is mainly determined by the donor concentration in the highly doped $\mathrm{Al}_{0.3} \mathrm{Ga}_{0.7} \mathrm{As}$ material. Both silicon MOS field effect transistors and $\mathrm{GaAs}-\mathrm{Al}_{0.3} \mathrm{Ga}_{0.7} \mathrm{As}$ heterostructures have been used for measurements of the quantized Hall resistance and have yielded identical results.

\section{Experimental Data}

Fig. 3 shows a typical result of Hall effect and resistivity measurements on a twodimensional electron gas. $A$ $\mathrm{GaAs}-\mathrm{Al}_{0.3} \mathrm{Ga}_{0.7} \mathrm{As}$ heterostructure was used in this experiment, because a fully quantized energy spectrum, necessary for the observation of the quantized Hall resistance, is already obtained at a relatively low magnetic field: the cyclotron energy for electrons in $\mathrm{GaAs}$ is much larger than in silicon for the same magnetic field.

The Hall voltage $U_{H}$ and the voltage drop $U_{p}$ between the potential probes are measured under constant current conditions $(/=$ const.) as a function of the magnetic field applied perpendicular to the plane of the 2DEG. The oscillation in $R_{x}=$ $U_{p} / /$ originates from variations in the filling factor of the energy levels $E_{n j}$ in a magnetic field. Whenever $R_{x} \sim \rho_{x x}$ becomes zero, an integer number $i$ of energy levels $E_{n j}$ is occupied and the Hall resistance adopts the value $R_{H}=h / e^{2} i$. Maxima in $R_{\mathrm{x}}$ are observed at approximately half-filled energy levels.

The simple theory discussed before cannot explain the flat regions in the $U_{H}(B)$ measurements. For a fixed surface carrier density $n_{e}$ only at well defined magnetic field values $B_{i}=h n_{\mathrm{e}} / e i$ (see eq. 3 ) should the Hall resistance be expressed by $R_{H}=$ $h / e^{2} i$. A number of theoretical papers have discussed the phenomena of Hall steps and the authors conclude that the correct value $R_{H}=h / e^{2} i$ should be observed, only so long as the condition $\rho_{x x}=0$ is fulfilled.

As experimental results are always obtained at finite temperatures, where a thermal excitation of electrons across the energy gap is possible, the condition $\rho_{x x}=$ 0 is never exactly fulfilled and corrections to the value of the quantized Hall resistance could be necessary. However, the value of $\rho_{x x}$ can be strongly reduced by decreasing the temperature and increasing the magnetic field and all experiments demonstrate that at the present level of accuracy, corrections due to the finite value of $\rho_{x x}$ are unimportant.

Different national standards laboratories will be able to measure the quantized Hall resistance in the near future with a resolution of $10^{-8}$, and it remains to be seen whether even at this level of accuracy, the experimental results are reproducible and independent of the geometry and the microscopic details of the device used. 